\title{
On the number of Klein bottle types
}

\author{
Carlo H. Séquin \\ CS Division, University of California, Berkeley \\ E-mail: sequin@cs.berkeley.edu
}

\begin{abstract}
Different geometric realizations of topological Klein bottles are discussed and analyzed whether they can be smoothly transformed into one another and thus belong into the same regular homotopy class. Simple and distinct representatives for each of the four expected classes are introduced. In addition, novel and unusual geometries for Klein bottles are presented, some of them knotted, which may serve as proposals for large-scale sculptures.
\end{abstract}

Keywords: Klein bottle types; topology; regular homotopies; Klein knottles; combination of Möbius bands. Classification: 58B05

\section{Introduction}

A Klein bottle is a closed, single-sided mathematical surface of genus 2, sometimes described as a closed bottle for which there is no distinction between inside and outside. A canonical example of this surface is depicted in Figure 1a with its characteristic "mouth" at the bottom, where the "inside" of the surface connects to its "outside." However, if one consults Wikipedia or some appropriate math text, one can find Klein bottles that look radically different, such as the twisted loop with a figure- 8 profile (Figure 1b) or Lawson's [9] single-side surface of genus 2 (Figure 1c), which has been conjectured to minimize total bending energy for such a surface [8]. Probing further, one can also find the intriguing glass sculptures of Alan Bennet [3] (Figure 2a-f), or various forms of knotted Klein bottles presented at Bridges 2012 [15], some of which are depicted at the end of this article.

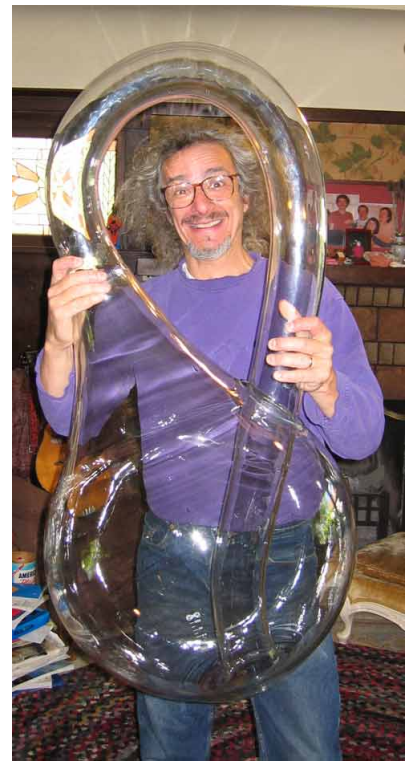

(a)

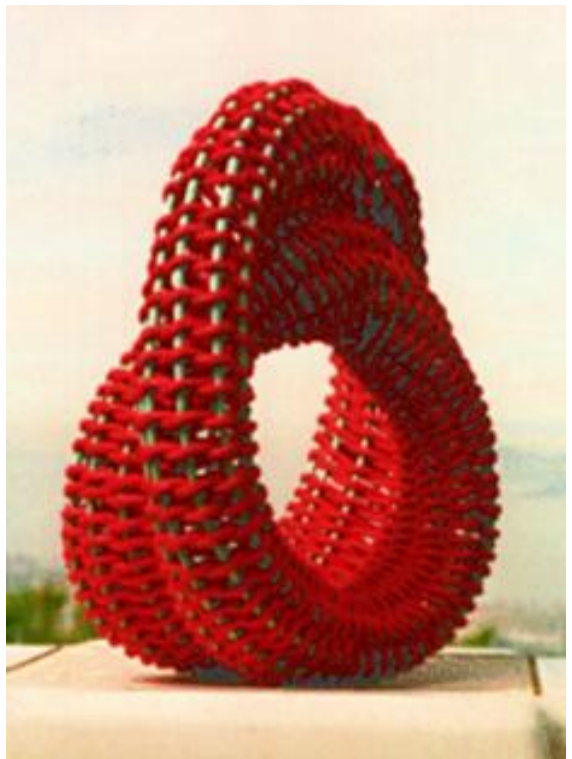

(b)

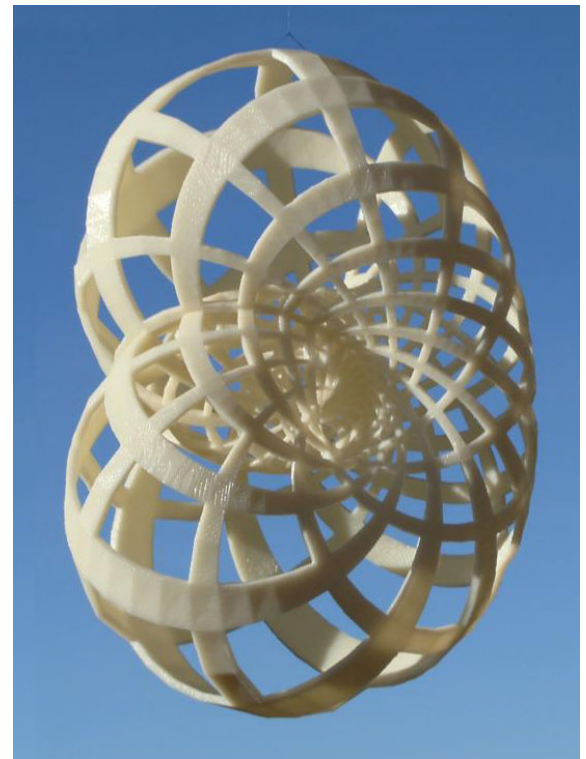

(c)

Figure 1: Klein bottles with different geometries: (a) Cliff Stoll with a very large classical Klein bottle (from [18] used by permission); (b) woven Figure-8 Klein bottle (Séquin 1997); (c) Lawson's minimumenergy surface approximated with a fused-deposition model (Séquin 2011).

Clearly, all these shapes are geometrically different. But how many of them are fundamentally different, if smooth deformations are allowed to transform one shape into another? If we ask mathematicians how many different types of Klein bottle there are, they will typically say: "Three or four - it all depends on 
whether the surface of the bottle is marked, e.g. decorated with a coordinate grid." To come to this conclusion, mathematicians allow smooth deformations that are called regular homotopies. These are transformations where a surface is allowed to pass through itself; but the surface may not be punctured, cut, or torn, and it must not assume any sharp creases or form any other singular points with infinitely sharp curvature. The precise conditions that a regular homotopy must satisfy are even more stringent [7][6][10], but those subtleties have no impact on the discussions that follow. For well-versed mathematicians, this paper may not break novel ground. All of the topological facts and insights are contained in papers such as [1][2][6][10]. The goal of this paper is to make these important findings accessible and understandable to a much broader audience, and to introduce representatives of the different Klein bottle classes in as symmetrical and easy-to-understand form as possible (Figure 13). To set the stage, we start with a discussion of the regular homotopy classes of annuli and of Möbius bands, which then enable the constructions of the three types of unmarked Klein bottles; two are chiral and mirror images of each other, and one is amphichiral. We then show that when surface markings are introduced, the latter class splits into two separate regular homotopy classes. We conclude with a presentation of several more complex Klein bottles and outline an analysis technique that should allow the reader to classify those contorted geometrical shapes as well as Bennet's sculptures (Figure 2).

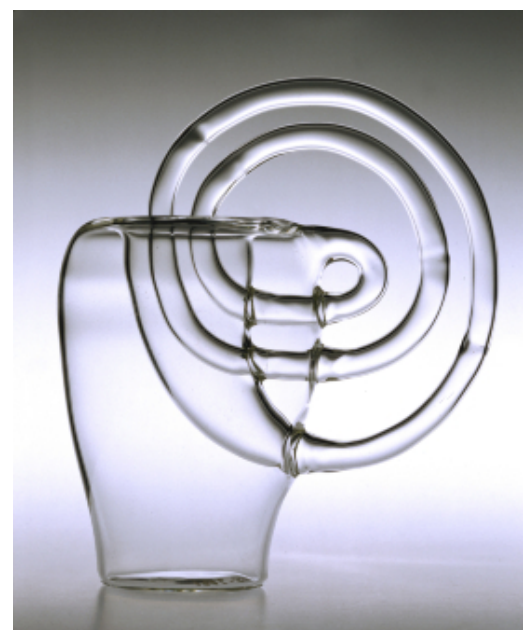

(a)

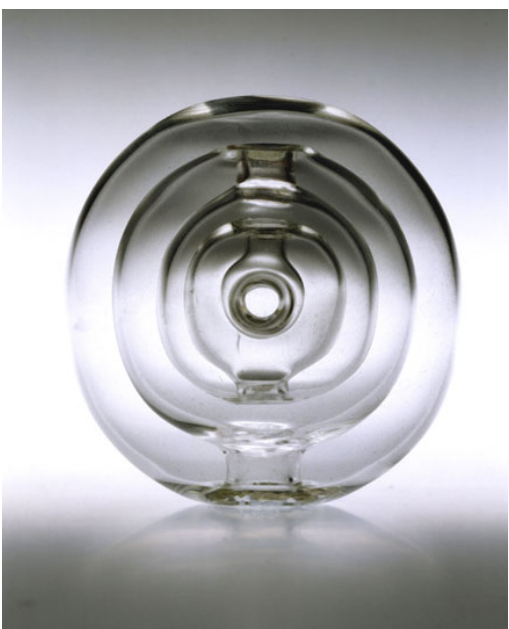

(b)

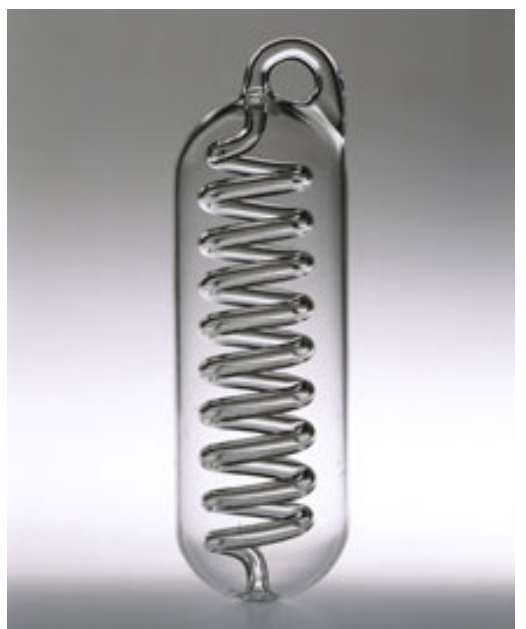

(c)

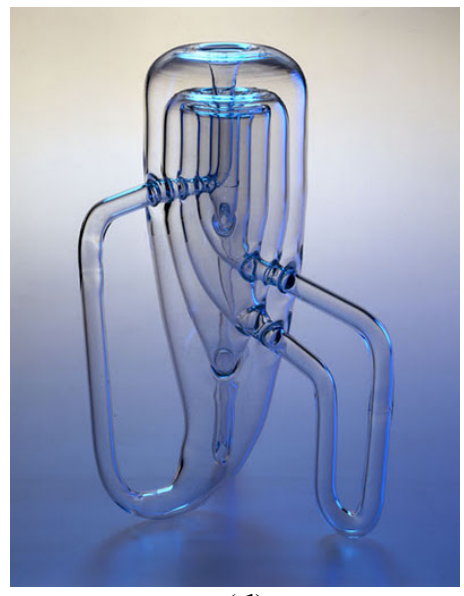

(d)

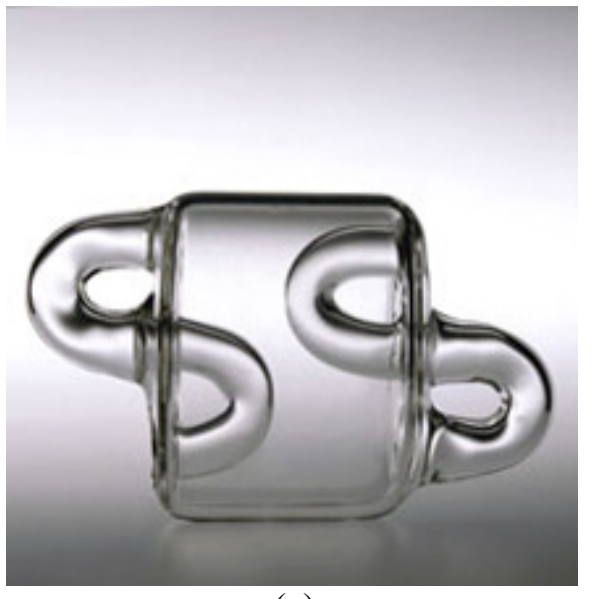

(e)

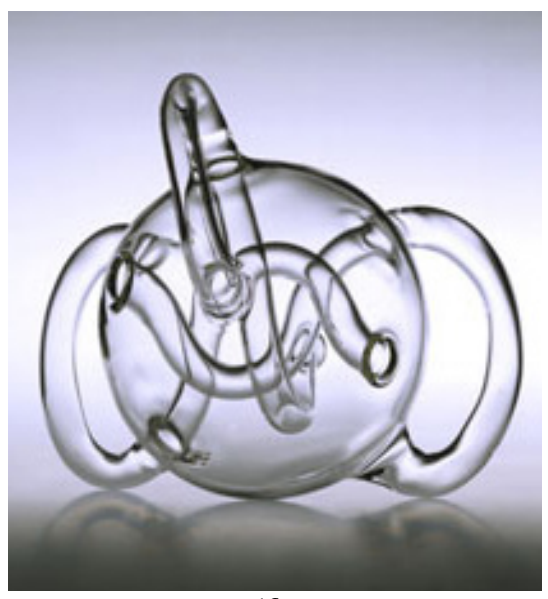

(f)

Figure 2: Glass sculptures by Alan Bennet: $(\mathrm{a}, \mathrm{b}, \mathrm{c})$ fancy Klein bottles exhibiting artistic geometry. More complex shapes: (d) three nested Klein bottles; (e) 2-handle Klein bottle; (f) 3-handle Klein bottle. All models are at the Science Museum / SSPL in South Kensington, UK (from [3] used by permission). 


\section{Regular homotopies of twisted bands}

We first analyze how many different types of twisted bands can be formed that cannot be transformed into one another with regular homotopies. We start with the rectangular piece of surface depicted in Figure 3a. Both sides are decorated with two chains of white and black arrow heads to provide a sense of directionality. The "front" face is also showing a rainbow colored background, while the "backside" exhibits a checkerboard pattern. Now we explore different ways of closing this band into a loop.

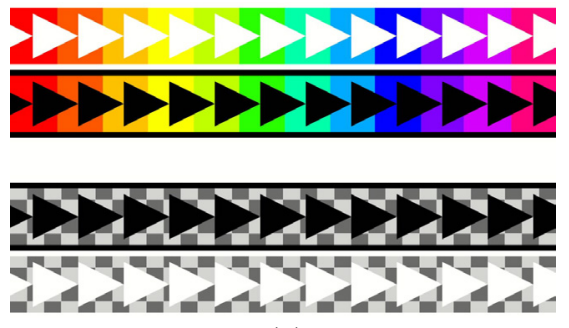

(a)

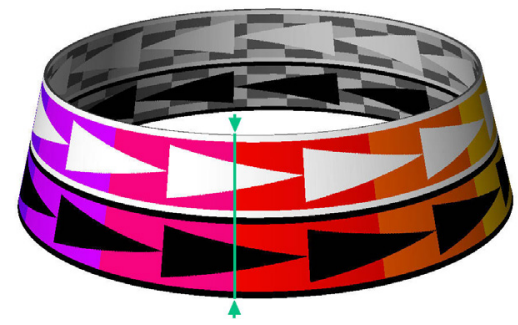

(b)

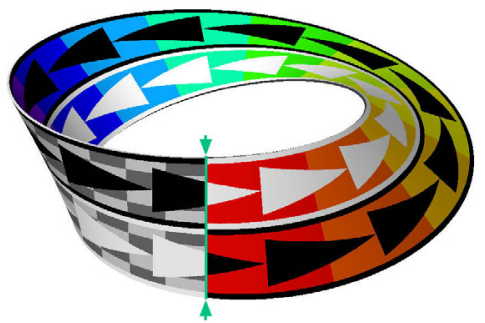

(c)

Figure 3. (a) Rectangular domain, front and back. (b) Merging the two short edges to form an untwisted annulus. (c) Closing the loop after a left-handed half-twist of $180^{\circ}$ has been applied to the band.

First we join the two short, vertical edges without imparting any twisting motion; this will form an untwisted annulus or generalized cylinder with two clearly distinct surfaces: one is rainbow colored and the other one is showing a checkerboard (Figure 3b). Alternatively, we could apply a half-twist of $180^{\circ}$ before joining the two ends (Figure 3c). This leads to a surface that is clearly quite different. At the juncture, the rainbow pattern joins the checkerboard; thus we can travel from one face to the other without having to climb over the rim or boundary of the surface. The surface is now said to be single-sided. It is a Möbius band. This is now a topologically different surface, and these two shapes cannot possibly be transformed into one another; to do so would require changing the appearance of the marked line in Figure $3 b$ to that in Figure $3 c$, i.e., changing the structure of the underlying surface

But there are more options. We could apply one or more full twists of $360^{\circ}$ before joining the two ends. These surfaces are then all two-sided. But are they all in the same regular homotopy class? Conversely, if we apply an odd number of $180^{\circ}$ half-twists, the surfaces will always be single-sided, but not all of them can be transformed into one another. Furthermore, we could let the band form a figure- 8 shape (Figure 4) or pass through multiple loops before we join the ends (Figure 5). Before we can classify all these many possible shapes, we have to understand how the apparent twist in the band is affected as we try to unwind a more complicated path through space into a simple loop with a planar, circular core curve (the center line of the band).

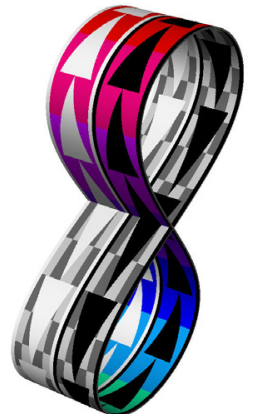

(a)

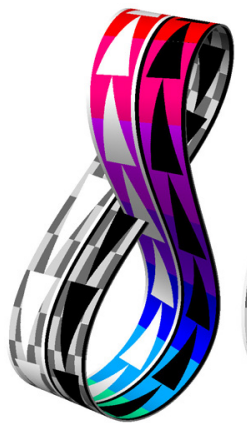

(b)

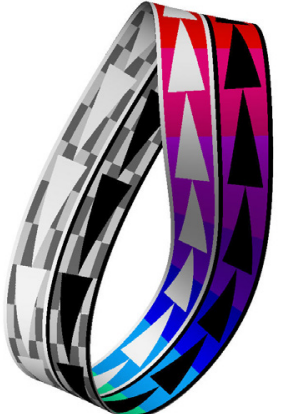

(c)

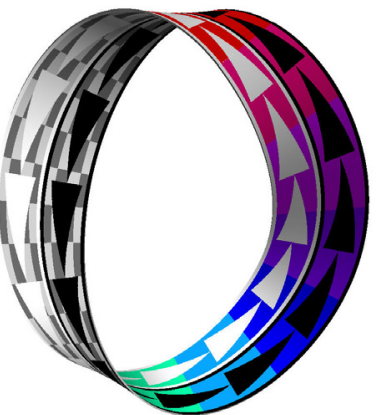

(d)

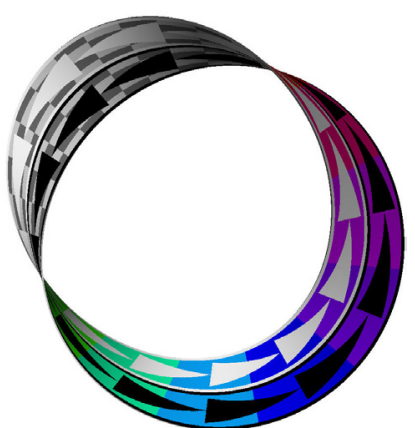

(e)

Figure 4. Unwarping a figure- 8 path: (a) starting shape; (b) warped space curve $90^{\circ}$ twist; (c) seriously warped curve, $180^{\circ}$ twist; (d) $320^{\circ}$ twist; (e) circular loop with $360^{\circ}$ twist. 


\subsection{Unwarping a figure-8 path}

Since we are not allowed to form any sharp creases or pinch-off points, we can only transform a figure- 8 path (Figure 4a) into a circular path by making a move through 3D space (Figures $4 \mathrm{~b}$-e). We start with an initially "untwisted" band, where the amount of twist is determined by the amount of extra rotation that needs to be applied to a rotation-minimizing frame in order to keep the band closed smoothly. As the initially planar, figure- 8 shaped core curve is deformed into a warped space curve, a steadily increasing amount of twist has to be added to keep the band smoothly closed. By the time the core curve has been opened up into the final, circular loop with again a planar core curve, a twist of $360^{\circ}$ has been imparted onto the ribbon (Figure 4e). Conversely, an undesirable twist of $\pm 360^{\circ}$ can be converted into a figure-8 sweep path. Furthermore, if we start from Figure 4e, first warp the band into a figure- 8 shape (Figure 4a), then continue the process by letting the band pass through itself, until the core curve exhibits again a perfectly circular loop, we find that the total twist in the band changes by exactly $720^{\circ}$. Thus the twist in any closed ribbon can readily be changed by $\pm 720^{\circ}$ by performing such a Figure- 8 Sweep Cross-over Move. This fact is closely related to Dirac's Belt Trick or Feynman's Plate Trick [5]. It also played an important role in a similar study [13][16] to find all the different regular homotopy classes of topological tori - two-sided, orientable surfaces of genus 1 . It turns out to be true in general that, topologically, the twist in any such band should be counted modulo $720^{\circ}$.

\subsection{Unwinding an extra loop}

If the initial configuration exhibits multiple loops (Figure 5a), we can unwind such a coil in the following way: One loop at a time is folded out and swung like an opening door through $180^{\circ}$ as is shown for the outer-most loop in Figures $5 \mathrm{~b}-\mathrm{d}$. The resulting figure- 8 configuration may still have multiple loops in one of its lobes (Figure 5d). It is interesting to note that this folding-out process by itself does not change the twist in that loop, even though it does change the azimuth and switch "inside" and "outside" of the moving ribbon segment. (The amounts of twist imposed on the upper and lower half of the moving loop due to the 3D space-warp of the corresponding core curve segments cancel each other out exactly.) However, the un-warping of the figure-8 lobe generated by the un-folding process (using the process depicted in Figure 4) will add a twist of $\pm 360^{\circ}$ as discussed above.

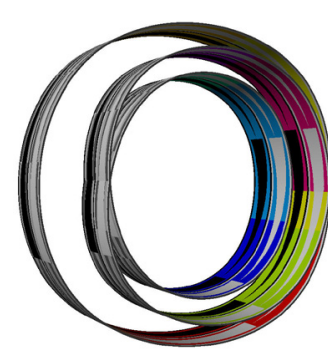

(a)

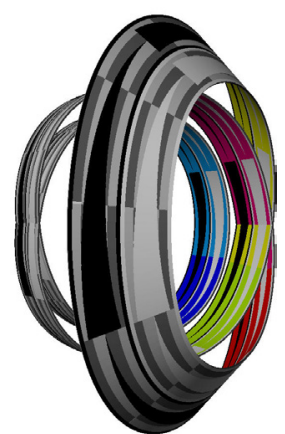

(b)

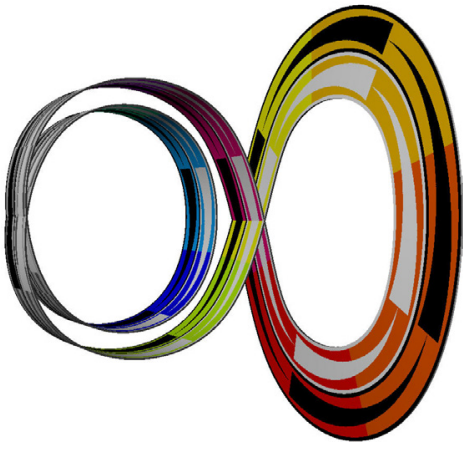

(c)

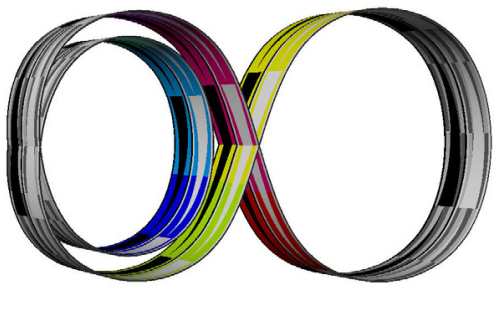

(d)

Figure 5. Unwinding an extra loop: (a) starting configuration with planar core curve; (b) $45^{\circ}$ worth of unfolding; (c) $90^{\circ}$ worth of unfolding; (d) final figure-8 loop, again with a planar core curve.

Of course, the starting configuration may be a more complex tangle, in which the ribbon may be twisting through three dimensions, may be knotted, or even self-intersecting. However, in the domain of regular homotopies, we are able to move portions of this ribbon smoothly through each other. We can thus readily untangle any knot and always bring the core curve into a planar, circular configuration. In this state we can now unambiguously determine the twist of the ribbon by recording how the surface normal of the ribbon rotates with respect to the Frenet frame of the core curve. If the integral amount of rotation exceeds the range $\left[-180^{\circ},+360^{\circ}\right]$ then we can reduce the amount of twist in increments of $\pm 720^{\circ}$ through the application of the figure-8 sweep cross-over move depicted in Figure 4. 
We thus find that for all closed, twisted bands there two topological equivalence classes (single-sided and double-sided) and two regular homotopy classes in each one of them. They are best represented by the untwisted annulus, designated AO (Figure 3b), by an annulus with $\pm 360^{\circ}$ of twist, labelled AT (Figure 4d), and by a left-twisting Möbius band, ML (Figure 3c) and its mirror image - a right-twisting Möbius band (MR). Note in particular, that none of the regular homotopies that we have examined so far can transform a Möbius band, say ML, into its mirror image (MR); in fact it can be proven formally that no regular homotopy can perform such a transformation [6] [7]. These important facts will now be used in the analysis of the number of Klein bottle types.

\section{Constructing Klein bottles}

A Klein bottle is a single-sided surface of Euler characteristic $\chi=V-E+F=0$ with no perforations or boundaries. It is essentially a single, contorted, self-intersecting "tube." It has been proven that in Euclidean space $\mathbf{R}^{3}$ no embeddings of Klein bottles are possible - only immersions, which exhibit some self-intersections [11]. Several different kinds of possible immersions will now be described through simple geometrical constructions of some basic Klein bottle types.

\subsection{The classical "inverted sock" Klein bottle}

We start with the rectangular domain depicted in Figure 6a. Since we want to construct only single-sided surfaces, we assume that this surface is made of a thin "glassy" material that carries the desired texture within, so that looking at it from either side will show mirror images of that pattern. The crucial point to forming a Klein bottle is to join opposite sides of this rectangle while observing the directionality indicated by the four arrows along the rectangle sides. For an illustrative construction, we first join the two longitudinal (horizontal) edges (with the cyan arrows) to form a generalized tube. This tube may have a simple circular cross section; alternatively it may assume a self-intersecting figure-8 profile, or even form a more complicated, multi-rolled tube. The difficult step is to now join the remaining open edge pair (brown arrows) with reversed directionality. Different types of tubes will require different deformations to accomplish this.

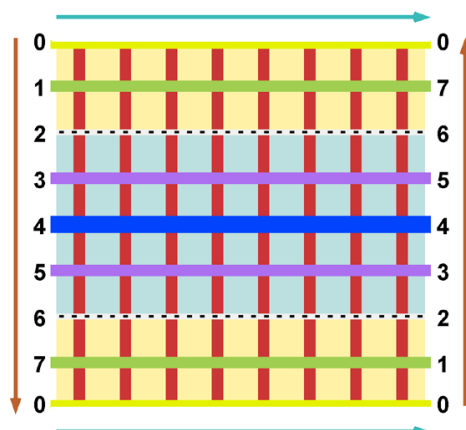

(a)

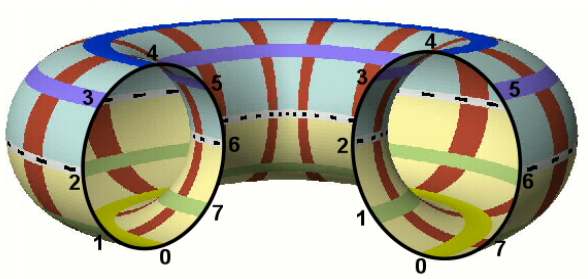

(b)

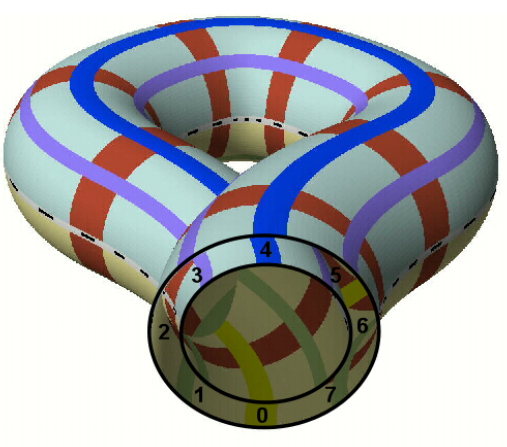

(c)

Figure 6: Construction of a Klein bottle: (a) its rectangular domain; (b) the domain rolled up into a tube; (c) the tube bent into a $\mathbf{J}$-formation with its two ends lined up so that labels match.

For the classical, "inverted-sock" Klein bottle with an O-profile, the closing of the loop is most conveniently done by narrowing one end of the tube and inserting it sideways into the larger end of the tube - forming a $\mathbf{J}$-shaped loop (Figure 6c); this configuration properly lines up all the numbered labels. Now the two concentric ends are merged by turning the smaller one inside out. This yields the classical "inverted sock" Klein bottle, named KOJ (Fig.7a). In this case, the lower half and the upper half of this merged loop independently form two Möbius bands of opposite handedness (yellow and blue) as shown in Figures $7 \mathrm{~b}$ and $7 \mathrm{c}$. We can denote this symbolically as: $\mathbf{K O J}=\mathbf{M R}+\mathbf{M L}$. 


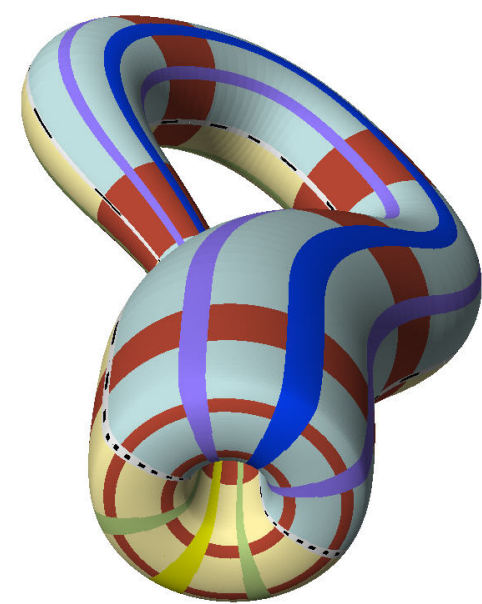

(a)

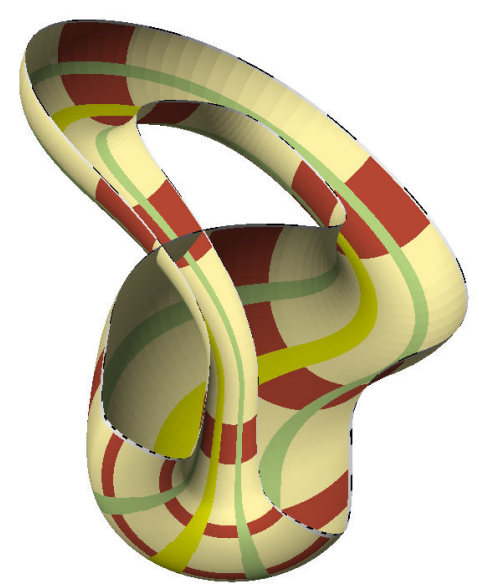

(b)

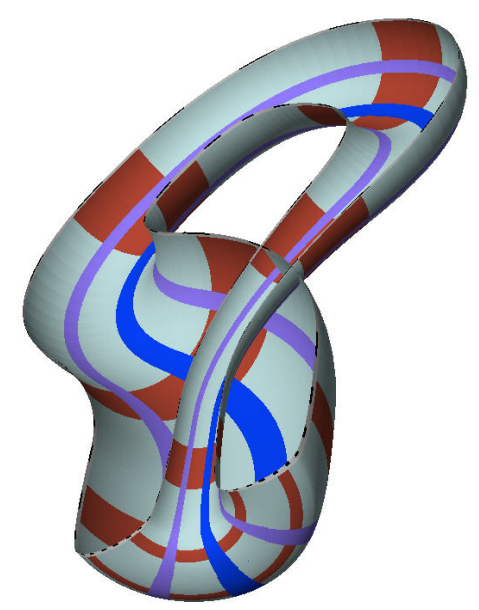

(c)

Figure 7: The ordinary "inverted sock" Klein bottle resulting from the construction in Figure 6: (a) the complete Klein bottle KOJ; (b) the lower half of it is a right-handed Möbius band (MR);

(c) the upper, left-handed Möbius band (ML) is shown flipped $90^{\circ}$ to the right.

\subsection{Figure-8 Klein bottles}

When merging the two horizontal edges marked by cyan arrows in Figure 6a to form an initial tube, we are not forced to form a round, circular $\mathbf{O}$-profile. Instead we may form a figure-8 cross section (Figure 8 a) or an even more complicated, multiply-rolled generalized cylinder, as was discussed for tori [14]. This will result in various Klein bottles, some of which may belong to different regular homotopy classes and thus cannot be smoothly deformed into the classical "inverted-sock" shape.

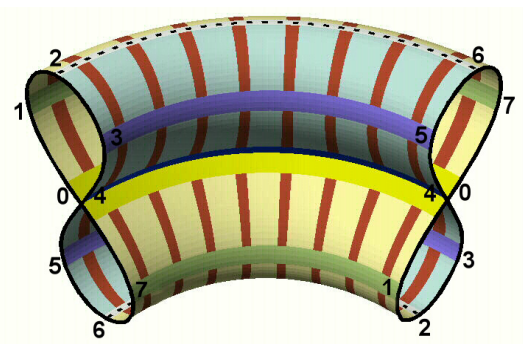

(a)

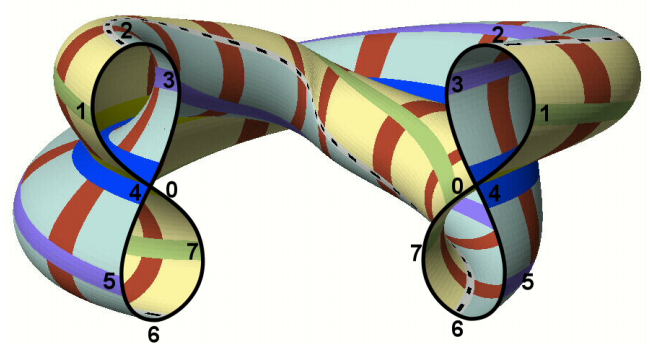

(b)

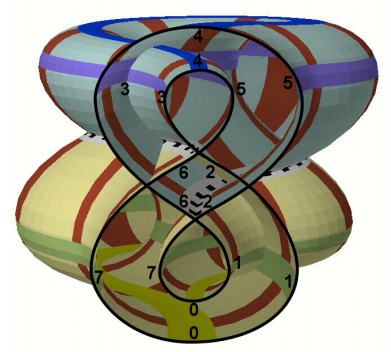

(c)

Figure 8: Constructions for figure- 8 Klein bottles: (a) figure- 8 tube; (b) the tube twisted through $180^{\circ}$ so that the two ends can be merged into a toroidal loop; (c) a new way to line-up the number labels.

For the tube with the figure-8 profile, there are a few different ways in which we can fuse the tube ends with the required reversed orientation, and they may result in different Klein bottles. For instance, we can bend the tube into a simple toroidal loop (an $\mathbf{O}$-shaped path) and give the figure-8 cross-section a $180^{\circ}$ torsional flip (Figure 8b); this flip can either be clockwise (right-handed: R) or counter-clockwise (lefthanded: $\mathbf{L}$ ). This will result in two figure-8 Klein bottles that belong to two different regular homotopy classes, which we call K8R-O (Figure 9) and K8L-O (Figure 10), respectively. To see that there can be no regular homotopy between these two types, assume that we remove the light yellow area from the rectangular domain shown in Figure 6a, and focus our attention on the remaining light blue area. This domain happens to be a right-handed Möbius band MR in the Klein bottle of type K8R-O (Figure 9), but is a left-handed Möbius band in the Klein bottle K8L-O (Figure 10); and, as mentioned above, no Möbius 
band can be transformed smoothly into its mirror image. The blue region and the yellow region of the texture domain (Figure 6a) appear in each of K8R-O and K8L-O; Figures 9 and 10 show these regions as individual Möbius bands, as well as joined together into Klein bottles. With this particular partitioning of the original domain, the pairs of Möbius bands that form the Klein bottles happen to intersect along their center lines. The domain could also be split in a different way, e.g., along a diagonal line; this would still partition the surface into two Möbius bands of equal chirality.

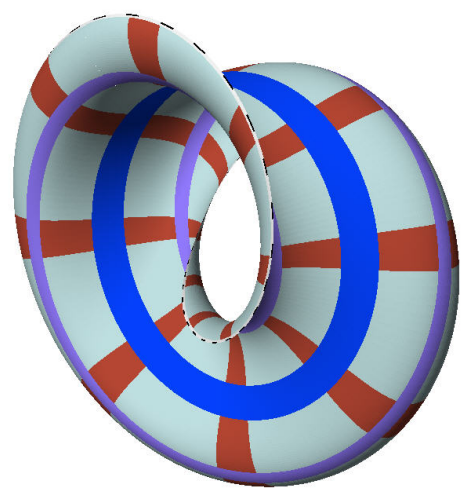

(a)

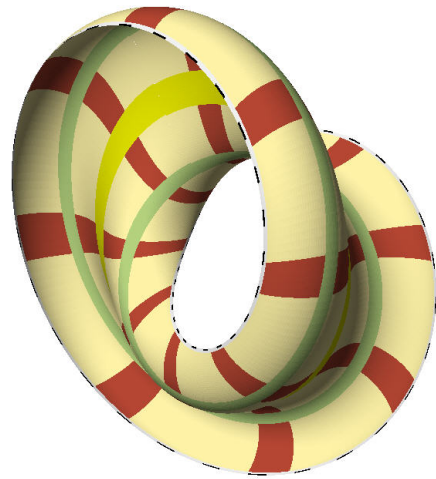

(b)

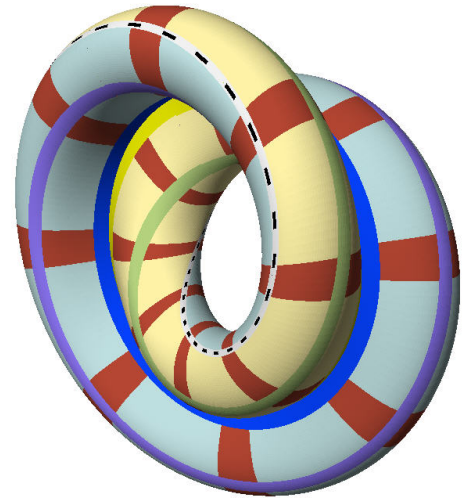

(c)

Figure 9: Two right-handed Möbius bands MR (a,b) form a right-handed Klein bottle of type K8R-O (c).

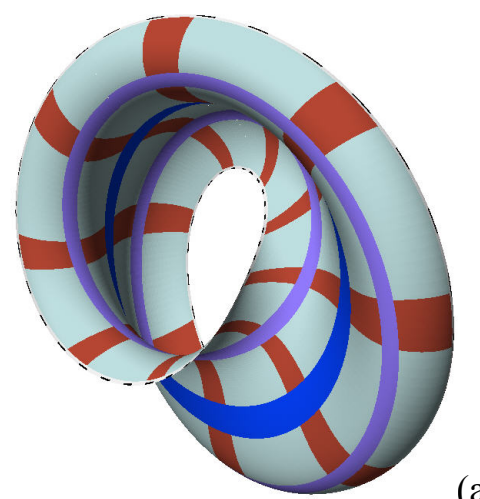

(a)

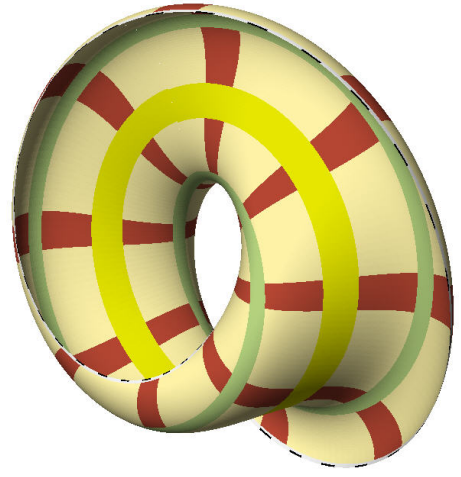

(b)

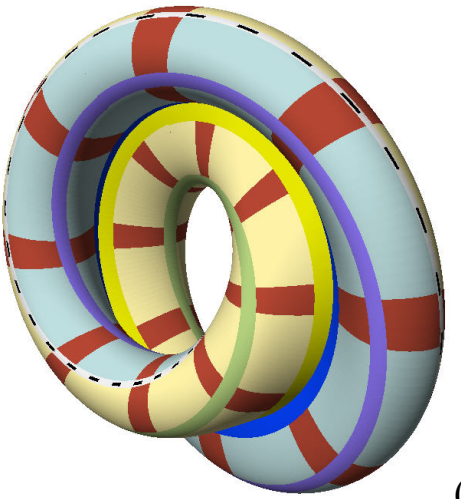

(c)

Figure 10: Two left-handed Möbius bands ML (a,b) form a left-handed figure-8 Klein bottle K8L-O (c).

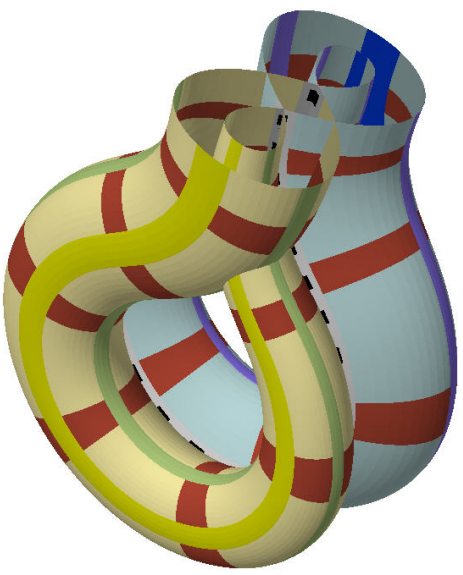

(a)

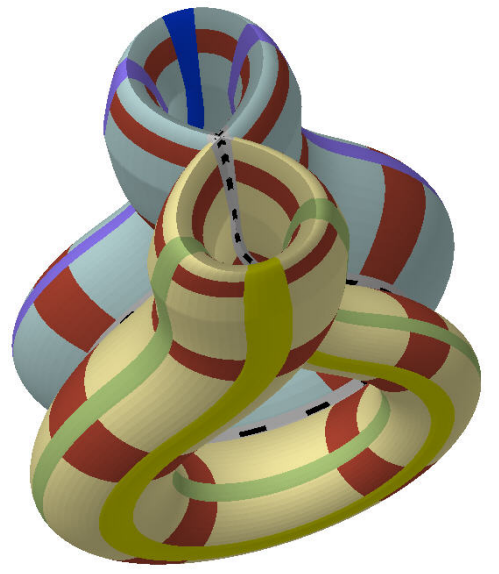

(b)

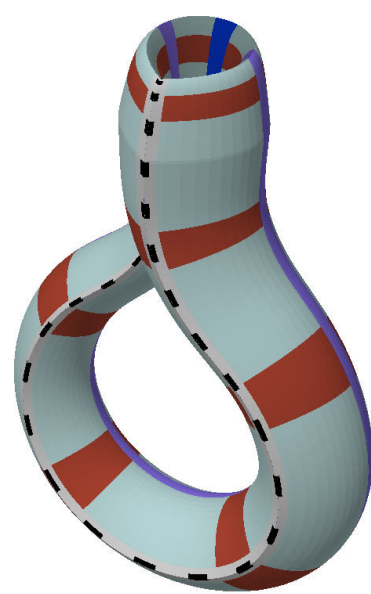

(c)

Figure 11: A Klein-bottle based on an "inverted double sock": (a) without end-caps to show the nesting of the tube profiles; (b) completed Klein bottle of type K8L-JJ; (c) one of its two ML Möbius bands. 


\subsection{Yet another form of Klein bottle}

Figure 11a shows a third way by which the 8-profile tube can be closed into a Klein bottle. This new type of Klein bottle is constructed by forming the same kind of $\mathbf{J}$-shaped sweep path as for the classical Klein bottle and fusing the two nested figure-8 profiles by turning one of them inside out. To do this smoothly, we use an asymmetrical figure-8 profile in which one lobe is larger than the other one. As this profile is swept from one end of the tube to the other one, the larger lobe shrinks and the smaller one grows, so that the end-profiles can be nested as shown in Figures $8 \mathrm{c}$ and $11 \mathrm{a}$. Now a nicely rounded, 8-shaped end cap can smoothly close off this Klein bottle (Figure 11b). I have not seen this particular Klein bottle depicted previously. It is rather special, since it does not just have a single self-intersection line like all the other models, but features two triple points. These triple points occur where the self-intersection line of the figure-8 cross section passes through the wall of the other tube near the mouth of the Klein bottle. Banchoff has shown that when triple points occur in Klein bottles, they must always occur in pairs [1].

A closer analysis of this Klein bottle reveals that its two constituent Möbius bands are of the same handedness, and it thus comes in two different chiral varieties; we name those K8R-JJ and K8L-JJ, respectively. We can split this Klein bottle cleanly along the closed self-intersection loop passing through both triple points to obtain two Möbius bands. Each such component on its own resembles an "inverted sock" Klein bottle shape with a sharp crease line, which itself exhibits a Möbius twist (Figure 11c). As we will see shortly, these type K8x-JJ Klein bottles are also in the same two regular homotopy classes as the K8x-O bottles, respectively.

\section{Marked Klein bottles}

A paper by Hass and Hughes [6] states (pg.103): Corollary 1.3 (James-Thomas): There are $2^{2-\chi}$ distinct regular homotopy classes of immersions of marked, closed surfaces of Euler characteristic $\chi$ into $\mathbf{R}^{3}$. This tells us there should be four distinct marked Klein bottle types that cannot be transformed smoothly into one another when surface markings are considered. Although [6] gives complete recipes of how to construct representatives of each type, I have found no paper that shows explicit pictures of all four types. The single-sidedness of these objects also makes it conceptually more difficult to visualize these shapes.

Since regular homotopy transformations cannot turn a left-twisting Möbius band into a right-twisting one [6] [7], we can immediately identify three types of Klein bottles that differ based on the handedness of the two Möbius bands from which they are composed, and which thus must belong to three different regular homotopy classes. The simplest representatives of the three classes are: K8L-O $=\mathbf{M L}+\mathbf{M L}$, $\mathbf{K 8 R}-\mathbf{O}=\mathbf{M R}+\mathbf{M R}$, and $\mathbf{K O J}=\mathbf{M L}+\mathbf{M R}$. The first two are chiral, and KOJ is amphicheiral (its own mirror image). These three classes are structurally different; they do not depend on any markings of the surface. This reveals an interesting difference to the world of tori [13][14], where there are only two structural classes: one formed by the three tori types TOO, TO8, and T8O, and the other one by $\mathbf{T 8 8}$ by itself.

Now, where do we find the $4^{\text {th }}$ anticipated type of Klein bottle? It cannot be composed of two Möbius bands of the same twistedness; because if we could find an additional bottle of this type, then its mirror image would be equally novel, and we would end up with five different types of Klein bottles. This also tells us that Lawson's Klein bottle, K8x-Lawson (Figure 1c), as well as the "inverted double sock", K8x-JJ (Figure 11b), both of which display $\mathrm{C}_{2}$-symmetry and an explicit handedness, cannot provide the sought-after $4^{\text {th }}$ representative and that there must be a way to transform them smoothly into the corresponding simpler twisted figure-8 loops, K8x-O (Figures 9, 10). Thus, the sought after type \#4 has to be composed of two Möbius bands of opposite handedness.

We can try to start from KOJ and somehow "switch" the two Möbius bands. But if we try this, and in this process flip the handle in Figure $7 \mathrm{a}$ to the right hand side, we can restore the original state by rotating the whole bottle through $180^{\circ}$ around the axis coming out of its mouth - unless we pay attention to the coloring! If the original KOJ is composed of a yellow MR and a blue ML, then its mirror image comprises a yellow ML and a blue MR, and no regular homotopy can deform one into the other. 


\subsection{Adding collars to a Klein bottle}

Topologists also have some other techniques for generating new representatives of a surface that then may fall into different regular homotopy classes. Hass and Hughes [6] start with a closed curve on the surface, turn it into a crease, and then add a fold that forms a kink or collar along that line. Let's see what results we get from grafting such collars to a Klein bottle. On Klein bottles, because of their singlesidedness, adding collars takes some extra care. To understand this we need to first discuss the various types of lines that can be drawn on a Klein bottle.

First, there are the meridians, shown as red bands in my surface texture. If we remove such a meridional band from a Klein bottle, the surface left over has the topology of an open cylinder with either an untwisted $\mathbf{O}$-profile or a (twisted) figure-8 cross section; these are in the same regular homotopy classes as the annuli AO and A8, respectively. Second, there are the center lines of the two Möbius bands, which are single-sided and either left- or right-twisting. Next, there are all the other longitudinal lines that run on either side of the Möbius core curves; they run twice around the loop before they close onto themselves, and they have an integer amount of twist. The removal of a small strip of surface along such a line will partition the Klein bottle into two Möbius bands. Finally there are diagonal lines that can be seen as connected combinations of the aforementioned lines. And, of course, there are trivial local loops that can be contracted to a single point; they are of no interest in our discussions and should be avoided.

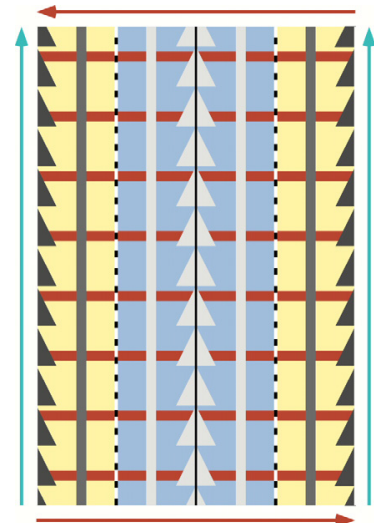

(a)

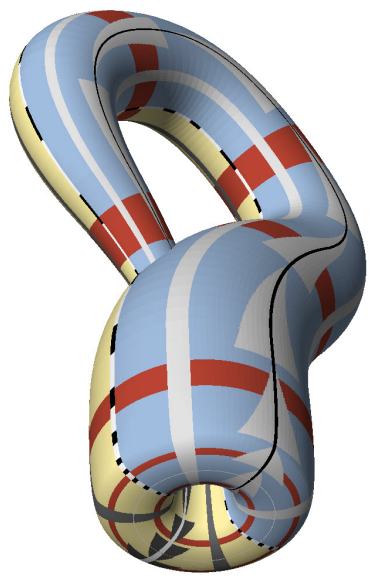

(b)

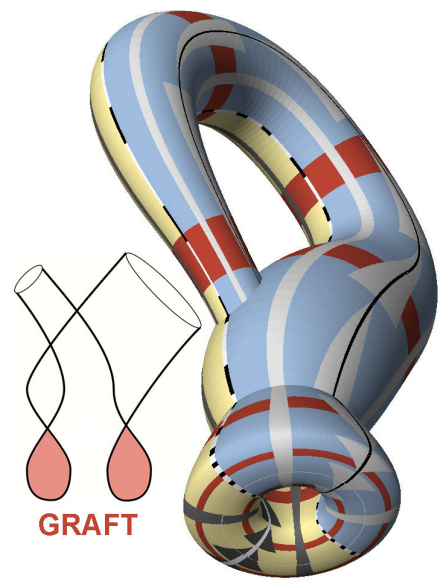

(c)

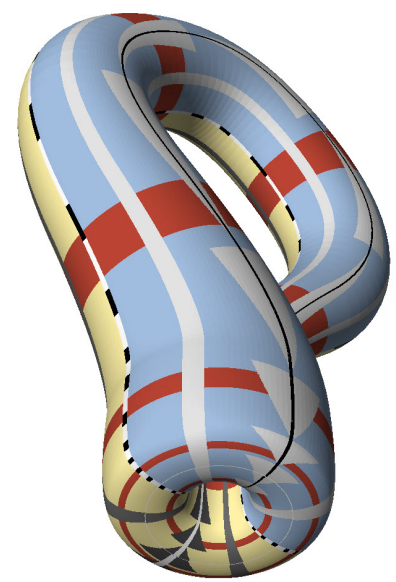

(d)

Figure 12: (a) New texture with longitudinal directionality; (b) classical KOJ with this texture applied; (c) KOJ with a collar grafted at the mouth; (d) KOJ with inflated left branch, eliminating the collar self-intersection, and resulting in a swap of the two colors and reversed longitudinal directionality.

We can graft a meridional collar anywhere along the tube forming the Klein bottle. In particular we can use the meridian at the rim of the mouth (Figure 12b), which then results in the collared structure KOJ-C shown in Figure 12c. The fold of this graft forces the tube to undergo an extra surface reversal and thus changes the directionality by which the directional texture (Figure 12a) passes through the mouth. While in Figure 12b the arrows seem to flow out of the mouth, in Figure 12c they flow into it. It turns out that we can easily eliminate the newly generated circular intersection line created by the grafted-on collar by inflating the left branch of the tube; we then simply obtain a Klein bottle in which the thin and the thick arms emerging from the mouth have been switched. But both Möbius bands now exhibit reversed handedness! Structurally, this makes no difference, since the original KOJ could simply be rotated $180^{\circ}$ around the mouth axis to coincide with the new shape. However, if we place a texture on the surface that exhibits directionality in the longitudinal direction or which colors the two Möbius bands differently, then the result is indeed in a different regular homotopy class. Here is an analysis of the twistedness of the various characteristic ribbons in response to the added meridional graft: The meridional ribbons are not affected. But the longitudinal Möbius bands, which pass through the grafted collar exactly once, 
experience an extra twist of $\pm 360^{\circ}$ which reverses their chirality; the left twisting Möbius band becomes right-twisting, and vice versa. This confirms that we have found a representative of the $4^{\text {th }}$ regular homotopy class for Klein bottles (Figures 12c and 12d); this is my preferred representation of this type.

We can also graft a fold onto a meridian of a Klein bottle with a figure-8 cross section. Such a collar travels on the outside for half the figure-8 and on the inside for the other half of the profile, and then closes smoothly onto itself. Again, the meridional bands are not affected. Again, each Möbius band crosses this fold exactly once and thus changes its twist by $360^{\circ}$, which reverses its twistedness. Thus, adding a figure-8 shaped M-graft will turn K8R-O into K8L-O and vice versa, but it does not generate a new regular homotopy class.

Adding a collar along a general longitudinal line that passes around the loop twice does not affect other longitudinal lines. It adds two reversing lobes to every meridian and thus does not affect their net twist. Such L-grafts thus do not change the regular homotopy class. A more complete discussion of all the collar-grafting options and how they convert Klein bottles from one regular homotopy class into another one can be found in [17].

\subsection{Change of surface parameterization}

For a torus, some discrete changes in the parameterization grid may yield a torus that belongs to a different regular homotopy class. In particular, the introduction of a Dehn twist [4] of $360^{\circ}$ in the meridional direction (M-twist) converts a torus of type TOO into TO8, and a longitudinal (or "equatorial") Dehn twist (L-twist) takes TOO into T8O. Thus we can try to apply all the surface reparameterizations that we have studied for the case of tori [13][14] and see how they affect the classifications of various Klein bottles. However, we already know that none of them can change the structural classification, since the effect of such re-parametrizations would be completely invisible on unmarked glass surfaces.

Again the situation is somewhat tricky on Klein bottles. Because of their single-sidedness, meridians cannot be assigned a consistent direction. This property rules out the possibility of a parameter swap that switches the roles between meridians and longitudes. On the other hand, it makes surface "eversion" trivial, since we can just shift the surface texture by one period in the longitudinal direction to have any surface normal point in the opposite direction. Also, as we have seen above, simply reversing the directionality in the longitudinal direction puts a KOJ Klein bottle into a different regular homotopy class; but such an L-reverse operation has no effect on the K8x types.

Introducing Dehn twist on Klein bottles also demands some extra care. Adding $360^{\circ}$ of meridional Dehn twist (M-twist) to a KOJ bottle is equivalent to adding a meridional collar: One feature can be turned into the other one by applying Dirac's belt trick [5] as illustrated in Figures 23 and 24 of [16]. Alternatively, we can remove any amount of twist in the handle of a KOJ type by swinging the handle through half the twist-angle around the mouth axis of the bottle [12]. On a marked KOJ bottle such a handle rotation will shift longitude curves out of alignment for all angles except for rotations in multiples of $180^{\circ}$. Keeping the coloring fixed on the outer, thicker arm near the mouth and swinging the handle around through $180^{\circ}$ transforms the Klein bottle into its mirror image and thereby reverses the chirality of both of its Möbius bands. The fact that each Möbius band changes its chirality as we apply an M-twist of $360^{\circ}$ can readily be understood when we contemplate what happens to the one end of a Möbius band, cut at a circular meridian, that is then forced to slide around this circle (which has a turning number of one).

With the same mental imagery we can also see that applying an M-twist to any bottle of type K8x does not change the twistedness of the two Möbius bands, since in this case the figure-8 shaped meridians have a turning number of zero.

For completeness we should also investigate the effects of Dehn twists along longitudinal and diagonal loops. It turns out that such operations must be disallowed on Klein bottles, since they lead to inconsistencies in the parameterization [17]. 


\section{Map of the regular homotopy classes of Klein bottles}

After having examined several different schemes for constructing Klein bottles and having identified the anticipated four different regular homotopy classes, we can now draw the complete map of all Klein bottle types, as was done for tori in 2011 [13][14]. Any possible Klein bottle, no matter how weird or how twisted, can be deformed by a regular homotopy into one of the four simple representatives depicted in Figure 13. In summary, we see that there are now three structural domains, one of which splits into two parts when surface parameterization is taken into account.
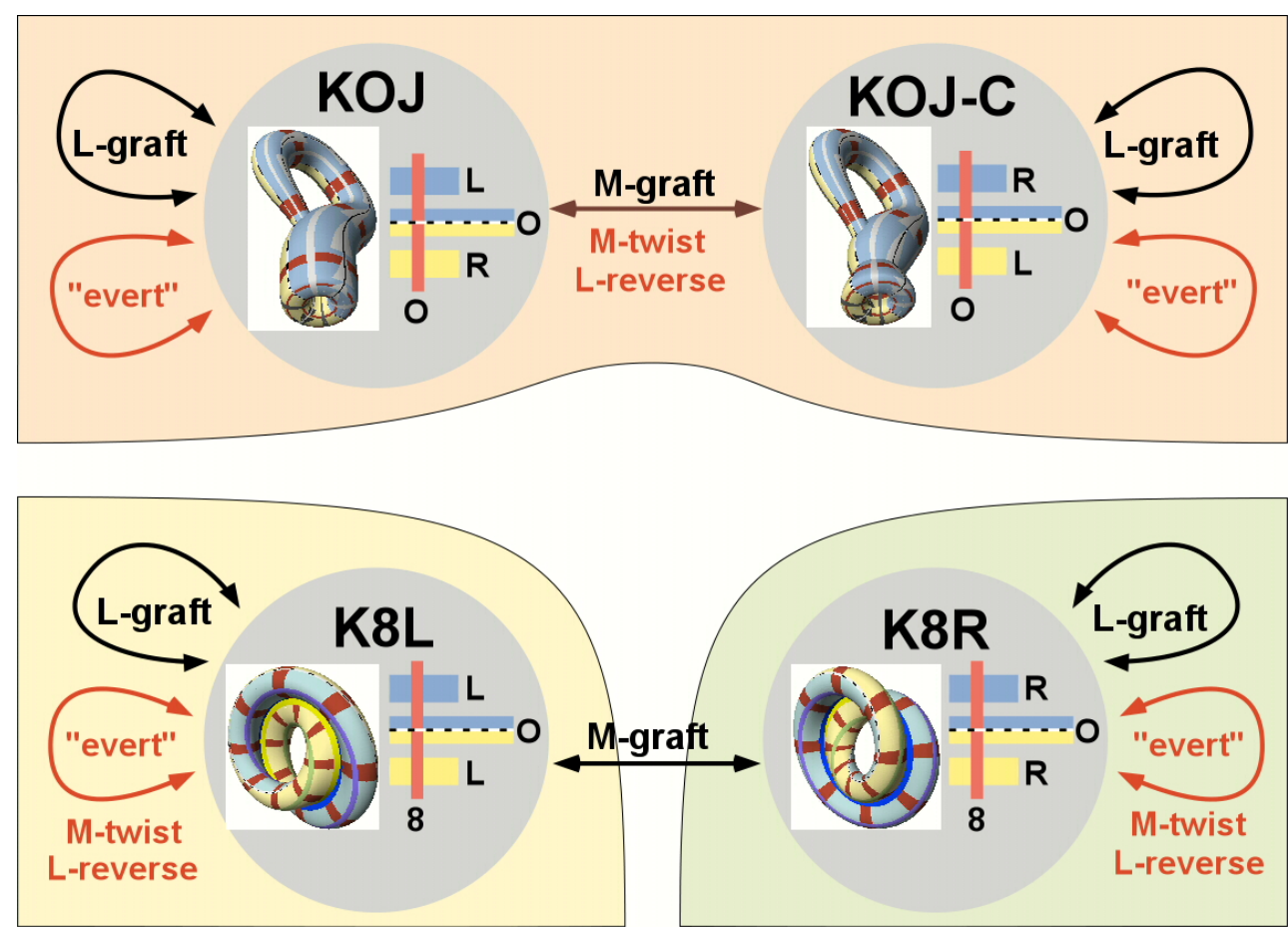

Figure 13: Complete map of the structurally different Klein bottle classes and of the effects of any surface re-parameterizations.

For each case, a small picture shows my preferred representative for that class, and, next to it, a symbolic representation of four characteristic ribbons on that surface. The red vertical stripe represents the meridional bands, and the horizontal stripes denote the center lines of the two Möbius bands as well as a general longitude ribbon that separates the two bands. The labels next to them tell whether that band is untwisted (O), or fully twisted (8), and whether a Möbius band is left-twisted (L) or right twisted (R).

Outside the grey circles there are some lines with double arrows, indicating various transformations that could be applied to a coordinate grid on the surface of the Klein bottle, and how they would affect the regular homotopy type of the transformed surface. As discussed above, the addition of a meridional collar or, equivalently, the addition of $360^{\circ}$ of meridional twist keeps the structure of the KOJ bottles the same, but changes the regular homotopy class for a marked surface; it either reverses the directionality in the longitudinal direction or it switches color between the two Moebius bands. Adding a meridional collar to a K8L bottle turns it into a type K8R, and vice versa. For a tube with a figure-8 profile this is not equivalent to adding a meridional Dehn twist; the latter leaves the K8x types unchanged. Also, a reversal of the longitudinal direction can readily be undone, by looking at a K8x-O toroid from the other side.

We also may wonder what the operation of surface eversion, which - somewhat surprisingly - kept all tori types within their own regular homotopy classes, would do for Klein bottles. It works the same way for Klein bottles, but here it is much easier to accomplish: we simply need to shift the coordinate grid 
one full period in the longitudinal direction, and every point will have moved to the "opposite" side of the surface.

On the other hand, the operation of parameter swap, which exchanges the roles of the meridional and longitudinal coordinate lines, is not possible for Klein bottles. All meridional lines always have an integral amount of full twists, while two of the longitudinal core curves exhibit the Möbius half-twists. No smooth continuous deformation can accomplish such a fundamental change.

\subsection{Surface analysis}

Figure 13 also tells how we can determine the types of the novel, fancy, twisted Klein bottles presented in the last section of this article. Those gridded models, as well as the glass sculptures by Alan Bennet [3] depicted in Figure 2, have no indication of directionality on their surfaces; thus we only need to figure out into which structural class they belong. First we make sure that the surface is indeed single-sided. For this we just need to find some arbitrary path that leads from a particular point on this surface to the "opposite side" at the same location. Next we check that the surface has Euler characteristic $\chi=V-E+F=0$; i.e., that it is essentially a single, contorted, self-intersecting "tube", possibly with some "inverted sock" turnbacks - but with no branching. If this "tube" cannot be readily identified, we would have to place a coarse graph of edge-connected vertices onto the surface and explicitly count the number of vertices (V), edges $(\mathrm{E})$, and facets $(\mathrm{F})$ in that mesh.

Once we have established that the surface indeed has the topology of a Klein bottle, the next crucial step is to find a Möbius ribbon on that surface. This may be a little tricky on surfaces such as the ones shown in Figure 1c or Figure 15b. In those cases we may want to trace out not just a single core curve, but accompany it by "parallel" side-lanes on either side. As we trace this "highway" over the surface until it joins itself again, we may find that all three lanes join themselves; this means we have traced out an orientable ribbon. We then need to continue our search until we find a "highway" loop in which the two side-lanes merge into each other, indicating a true Möbius ribbon. If we then "subtract" that ribbon from the surface, what is left over will form another Möbius band. We now determine the twistedness of both of these two Möbius bands, and this will define the regular homotopy structure of this Klein bottle as already pointed out in Section 4: $\mathbf{M L}+\mathbf{M L} \rightarrow \mathbf{K 8 L} ; \mathbf{M R}+\mathbf{M R} \rightarrow \mathbf{K 8 R}$, and ML+MR $\rightarrow$ KOJ.

To determine the twistedness of a space curve embedded in a complicated surface, I have had good success with the following method: Try to model in 3D space a thin ribbon made from stiff paper, so that it conforms to the Möbius band found on the surface in question (which may exist in the form of a 3D model or simply as a 2D rendering). When the paper strip has been shaped along the whole pathway of the ribbon, tape its two joining ends together, and then try to open it up into a circular loop in which the amount of twisting can readily be determined. In more complex cases, such as those depicted in Figure 14 , the paper strip may actually be knotted. In this case you will have to cut and re-join segments of this strip to untangle the knot; make sure that you preserve the alignment of the ends of the paper strip near those temporary cuts, so that the built-in twist is not altered. The total amount of twist observed in this circular loop can then be reduced modulo $720^{\circ}$ to fall into the interval $\left[-180^{\circ},+360^{\circ}\right]$ to make classification easy.

It is quite possible that when we search for that first Möbius core curve, we might not stumble upon the most direct closure for such a curve and find instead a line that makes an additional meridional loop around the "handle" of the Klein bottle, thus following some "diagonal" line. This may impart an extra twist of $360^{\circ}$ on such a ribbon and thus may reverse the chirality of that Möbius band. Would this not lead to a different classification? - No, it does not! This particular choice of a first Möbius band would also force the same extra meridional loop onto the second Möbius band. Thus in the case of a KOJ bottle, both Möbius bands would experience that extra twist and the resulting reversal of their chirality; thus the structural classification would remain unchanged. In the case of the K8x Klein bottles, the meridional band has a turning number of zero, and extra meridional detours will not add any twist to the Möbius bands; again, the classification remains unchanged. 


\section{Visualization models and sculptures}

Mathematical visualization models are important to render math insights understandable to a much broader audience than to mathematicians trained in topology and group theory. Making such visualization models as clear and unambiguous as possible is itself an art. Making them also aesthetically pleasing will entice a larger audience to pay attention and to study them. In some cases, well-conceived math models may even serve as inspiration for "constructivist" sculptures. Below I show some maquettes of gridded models of Klein bottles that might be turned into large sculptures by assembling them from I-beams or from tubular elements.

Figure 14 depicts two knotted versions of Klein bottles. The first one is a twisted figure-8 loop; but in this case the loop undergoes three flips of $180^{\circ}$, and the sweep path is closed into a trefoil knot. Figure $14 \mathrm{~b}$ depicts a different kind of trefoil knot; this one is composed of three interlinked segments of the classical "inverted sock" type of Klein bottle. A surface reversal takes place at each one of the three Klein bottle mouths. Thus, as long as there are an odd number of such surface reversals, the result will be a Klein bottle - rather than a warped self-intersecting torus. I like to call such knotted variants of Klein bottle shapes Klein-Knottles [15].

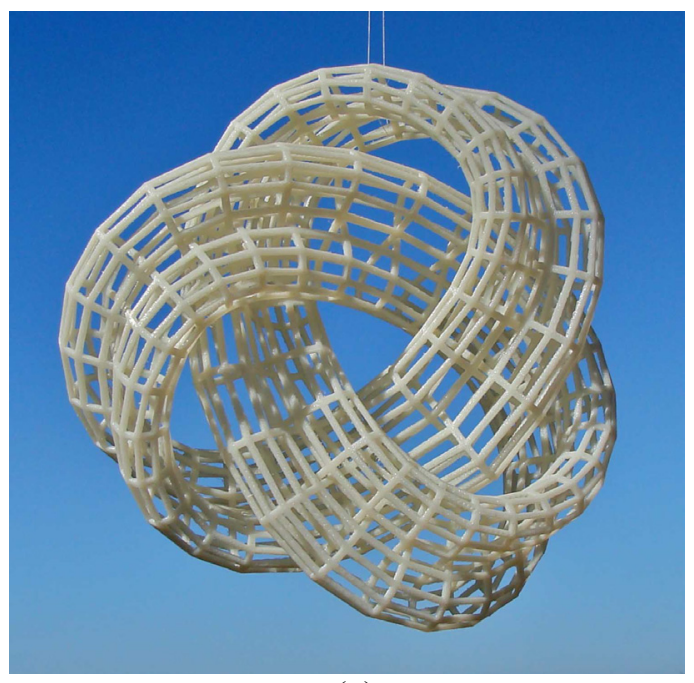

(a)

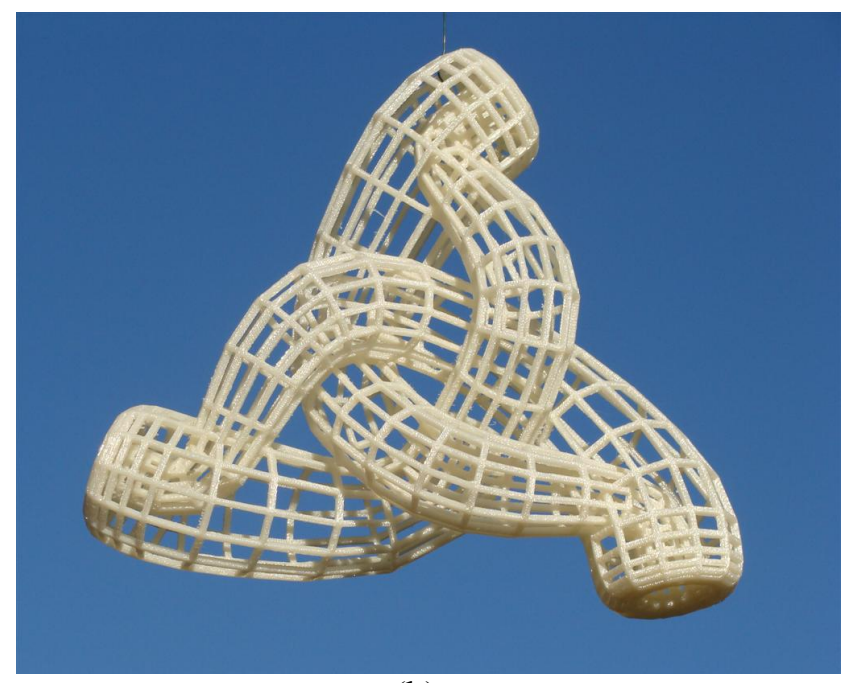

(b)

Figure 14: Maquettes of knotted Klein bottle sculptures: (a) Trefoil knot with figure-8 profile;

(b) trefoil formed by three Klein bottle sections of type KOJ.

Figure 15 displays some novel, more radical shapes for Klein bottles. First there is a gridded version of the "inverted double sock" Klein bottle, K8L-JJ, already introduced in Figure 11b. This gridded, "semitransparent" model allows a better inspection of how the figure-8 cross section turns inside out at the Klein bottle mouth and then morphs from one asymmetrical configuration to another one, in which the uneven sizes of the two lobes are reversed. As in the classical Klein bottle, the figure-8 profile mouth also accomplishes a surface reversal. Thus any un-twisted configuration with an odd number of such reversals will topologically form a Klein bottle. However, the figure-8 profile gives us another degree of freedom. It allows us also to accomplish a surface reversal by giving the figure-8 tube a $180^{\circ}$ flip - or an odd multiple thereof. These two mechanisms of surface reversals can be combined: We may use an even number of $180^{\circ}$ flips together with an odd number of Klein bottle mouths, or, alternatively, an even number of turn-back mouths together with an odd number of flips. Figure $15 \mathrm{~b}$ displays a very "compact" Klein bottle geometry, K8R-ZZ, with two Klein bottle mouths, combined with an overall twist of $180^{\circ}$. This twist is applied in two portions of $90^{\circ}$ each, as the figure-8 profile travels once up and down a portion of the $z$-axis. In one of these two passes, the profile also morphs into its twin shape, in which the sizes of the two lobes are exchanged. 


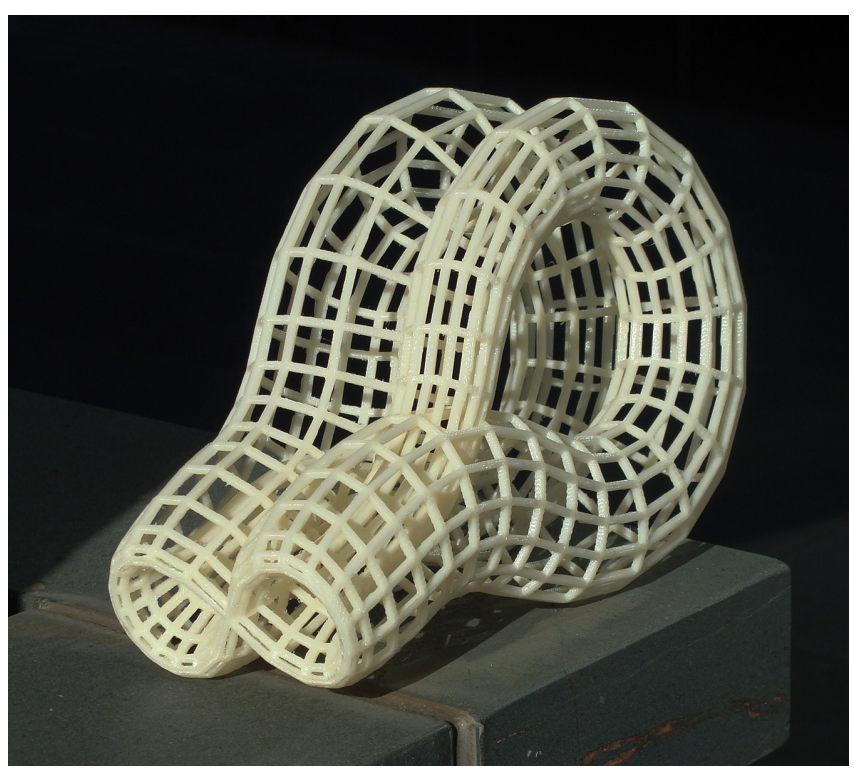

(a)

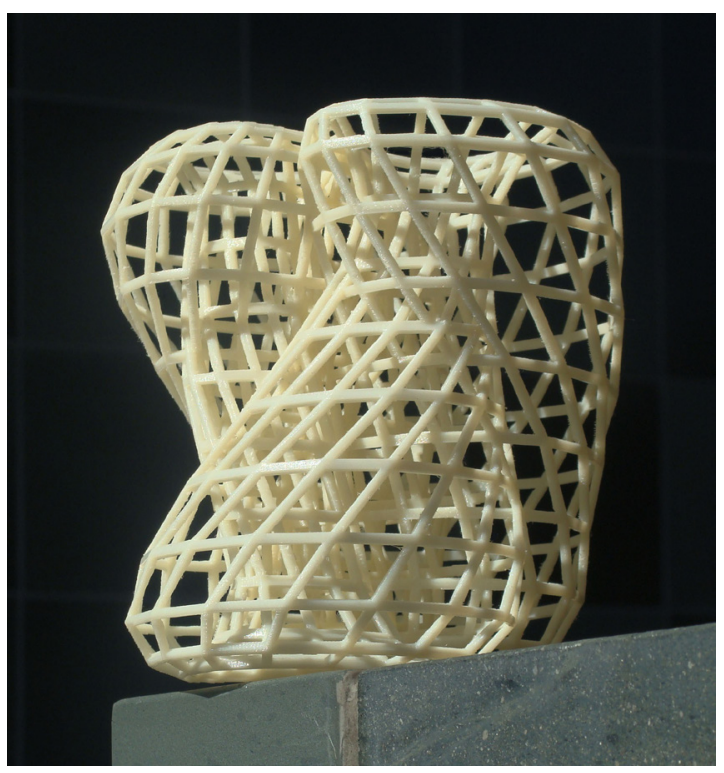

(b)

Figure 15: Unusual, heavily self-intersecting Klein bottle structures: (a) The left-turning K8L-JJ;

(b) a right-turning Klein bottle with figure-8 profile and a zig-zag sweep path, K8R-ZZ.

\section{Conclusion}

For most people who learn of the existence of Klein bottles, even if it is just the most frequently depicted "inverted-sock" geometry, these are fascinating objects. When one learns that there are several different Klein bottles that are not just smooth deformations of one another, these surfaces become even more interesting. Next, one may come across somewhat contradictory statements that claim that there are three or four "different" Klein bottle types - but typically without any explicit pictures of what these types may look like, and now one faces a really intriguing puzzle. Unfortunately, the mathematics literature that contains precise answers to all these issues is of a very technical nature, and thus the answers to these interesting questions have remained inaccessible to many potentially interested readers. I hope that this article sheds some light on these issues and further expands the catalogue of intriguing geometries that constitute topological Klein bottles.

\section{Acknowledgements}

I would like to express my thanks to Dan Asimov, Rob Kusner, and to Matthias Goerner for insightful and constructive comments on preliminary versions of this paper. I am truly indebted to the two anonymous reviewers whose extensive comments helped me to reduce ambiguous or misleading wordings and to enhance the clarity of the presentation. Their suggestions also encouraged me to provide better and more detailed explanations, and in this process I gained a deeper understanding of some of the underlying mathematical concepts.

This work was supported in part by the National Science Foundation (NSF award \#CMMI-1029662 (EDI)). 


\section{References}

[1] T. F. Banchoff, Triple points and surgery of immersed surfaces. Proc. Amer. Math. Soc. 46, pp 407413, 1974.

[2] T. F. Banchoff and N. H. Kuiper, Geometrical class and degree for surfaces in three-space. J. Diff. Geom. 16, pp 559-576, 1981.

[3] A. Bennet, Surface Model. - http://www.sciencemuseum.org.uk/objects/mathematics/1996-544.aspx (Feb. 2013).

[4] Dehn Twist: - http://en.wikipedia.org/wiki/Dehn_twist (Feb. 2013).

[5] Dirac's belt trick; Feynman's plate trick: - http://en.wikipedia.org/wiki/Plate_trick (Feb. 2013).

[6] J. Hass and J. Hughes, Immersions of Surfaces in 3-Manifolds. Topology, Vol.24, No.1, pp 97-112, 1985.

[7] M. W. Hirsch, Immersions of manifolds, Trans. Am. Math. Soc. Vol.93, pp 242-276, 1959.

[8] R. Kusner, Comparison Surfaces for the Willmore problem. Pacific J. Math. Vol.138, No.2, pp 317345, 1989.

[9] H. B. Lawson, Complete minimal surfaces in $S^{3}$. Ann. of Math., Vol.92, pp. 335-374, 1970.

[10] U. Pinkall, Regular Homotopy Classes of Immersed Surfaces. Topology, Vol.24, No.4, pp 421-434, 1985.

[11] H. Samelson, Orientability of hypersurfaces in $R^{n}$. Proc. Amer. Math. Soc. Vol.22, pp 301-302, 1969.

[12] C. H. Séquin, Twisted Prismatic Klein Bottles. The American Mathematical Monthly, Vol.87, No.4, pp 269-277, 1980.

[13] C. H. Séquin, Tori Story. Bridges Conf. Proc., pp 121-130, Coimbra, Portugal, July 27-31, 2011.

[14] C. H. Séquin, Torus Immersions and Transformations. UCB Tech Report (EECS-2011-83).

[15] C. H. Séquin, From Möbius Bands to Klein-Knottles Bridges Conf. Proc., pp 93-102, Towson, MD, July 25-29, 2012.

[16] C. H. Séquin, Topological tori as abstract art. Journal of Mathematics and the Arts, Vol.6, No.4, pp 191-209, 2012.

[17] C. H. Séquin, Regular Homotopies of Low-genus Non-orientable Surfaces - Revised and extended version. UCB Tech Report (EECS-2013-21), March 2013.

[18] C. Stoll, ACME Klein Bottles. Home page. - http://www.kleinbottle.com/index.htm (Feb. 2013). 